\title{
Place de l'autoconservation de sperme chez l'homme : Analyse d'une étude rétrospective
}

\author{
C. BARTHELEMY
}

CECOS Centre-Ouest, CHU Bretonneau, 37044 Tours Cedex

\section{RESUME}

L'autoconservation de sperme avant tout traitement stérilisant a pris un essor particulièrement important du fait des nouvelles techniques d'AMP.

Nous présentons ici les résultats d'une enquête rétrospective des conservations effectuées au CECOS de Tours de 1980 à 1990 et de leur devenir, en la comparant avec l'enquête nationale de la Fédération des CECOS.

Dans cette étude sont présentés, l'évolution de l'activité d'autoconservation, le profil de la population concernée, les caractéristiques spermatiques des éjaculats, et le devenir du sperme mis en banque avec son utilisation éventuelle.

Comme dans l'enquête nationale il est mis en évidence à la fois une augmentation des demandes, du pourcentage de spermes conservés par rapport aux demandes, et de l'incidence du cancer du testicule. L'atteinte du sperme est significativement plus importante dans les cancers testiculaires et les résultats des AMP dépendent de la qualité du sperme après congélation.

Avec l'avènement de la fécondation assistée, l'autoconservation de sperme est, en l'absence de protection efficace de la spermatogenèse, une obligation de moyens.
Mots clés : Autoconservation de sperme, AMP, fertilité, maladie de Hodgkin, cancers du testicule, cancers.

\section{INTRODUCTION}

L'amélioration du pronostic des cancers et l'allongement de l'espérance de vie soulève le problème de la fertilité ultérieure de patients jeunes en âge de procréer recevant un traitement anticancéreux. Le retentissement de ces traitements chimio et/ou radiothérapiques se situe à deux niveaux :

- possibilité d'une atteinte de la spermatogenèse entraînant une stérilité temporaire ou définitive iatrogène $[2,8]$ mais qui peut être aussi imputable à la pathologie elle même [10],

- risque d'anomalies dans la descendance après récupération éventuelle [1].

L'autoconservation de sperme avant tout traitement potentiellement stérilisant offre en France dans les CECOS depuis 1973, la possibilité de conserver le sperme longtemps sans altération de sa qualité et peut permettre de préserver des possibilités ultérieures de procréation $[3,4,12]$. Encore récemment cette possibilité était discutée étant données les altérations spermatiques que présentaient avant congélation certains patients [6]. Cette possibilité a pris à l'heure actuelle un essor particulièrement important et ses applications s'élargissent du fait des nouvelles techniques d'assistance médicale à la procréation [11]. Est présentée ici une 
étude rétrospective de ces autoconservations et de leur devenir, effectuée sur 11 ans au CECOS de Tours, dans le cadre d'une enquête nationale multicentrique diligentée par la Fédération Française des CECOS (Andrologie, 1996, v 6, nº 3., 301-310.

\section{MATERIEL ET METHODES}

Etude des dossiers des patients sur 11 ans (1980-1990) avec leur devenir au 1/01/95. Les données avaient été recueillies grâce à un dossier type mis au point au CECOS Necker. Les patients concernés ont eu recours à une conservation avant traitement, seuls les patients avec un sperme effectivement mis en garde et un dossier informatif au niveau biologique et médical ont été pris en compte.

L'exploitation des données a été réalisée sur Excel et Statview. Les valeurs sont données en moyenne \pm S.E.M. ou en pourcentage et les comparaisons de moyennes effectuées par Anova, tests non paramétriques et test de student.

\section{RESULTATS}

Sur la période 1980-1990, 210 dossiers seulement étaient réellement exploitables.

\section{Evolution de l'activité et des indica- tions d'autoconservation}

Les demandes vont en augmentant régulièrement depuis 1979 , les indications principales étant les cancers testiculaires $(76=$ $36,2 \%)$, les lymphomes $(97=46,2 \%: 81=$ $38 \%$ sont des maladies de Hodgkin), les autres cancers ne représentent que $17,6 \%$ des indications. Ces résultats sont identiques à ceux de l'enquête nationale. Le type histologique des cancers testiculaires est une donnée souvent méconnue, $40,8 \%$ dans notre centre versus $71 \%$ dans l'enquête nationale. Parmi ceux pour qui le diagnostic est précisé, $57 \%$ sont des sémi- nomes et $43 \%$ des tumeurs non séminomateuses, cette proportion reste stable au fil des années.

Il faut noter toutefois que si on étudie toutes les autoconservations réalisées depuis 1979 jusqu'à fin 1995, sur 491 spermes congelés pour les deux indications principales on note une proportion en constante augmentation des cancers testiculaires $(49 \%)$ alors que les maladies de Hodgkin restent stables depuis 1985 (38\%) (Figure 1).

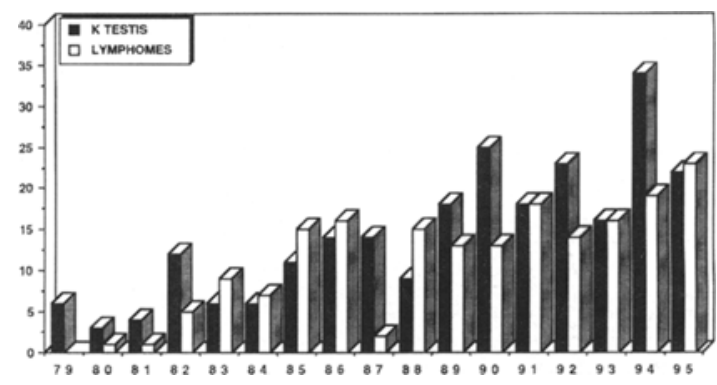

Figure 1 : Autoconservation : Evolution des demandes pour cancers testiculaires et lymphomes de 1979 à 1995.

De plus le pourcentage de spermes effectivement congelés augmente de manière significative depuis quelques années, si on exclut les azoospermies et les échecs de recueil (5 à 10\%) (Figure 2).



Figure 2 : Autoconservation : Evolution des demandes et spermes effectivement conservés en tenant compte ou non des axoospermies. 
La mise en œuvre de la congélation a toujours eu lieu avant tout traitement chimio et/ou radiothérapique dans notre centre.

\section{Profil de la population concernée}

- L'âge moyen des patients est stable au fil du temps (16 à 46 ans).

Il y a une différence significative entre les âges des patients atteints de cancers testiculaires ou de lymphomes $(p<0,0004)$, fait non retrouvé dans l'enquête nationale (Figure 3 ), mais il n'existe pas de différence intra groupes en fonction du type de la tumeur.

- L'analyse de la situation maritale sur la période étudiée, connue pour 183 dossiers $(87,2 \%)$, montre que $64,5 \%$ vivent en couples (dont $24 \%$ de concubins) versus $60 \%$ dans l'enquête nationale, seuls $35 \%$ des hommes sont célibataires (Figure 4).

- L'analyse de la paternité, connue dans $67 \%$, des cas montre que les patients atteints de cancers testiculaires sont plus souvent déjà pères mais la différence n'est pas significative (Figure 4).

\section{Caractéristiques des éjaculats conservés (Tableau 1)}

Toutes pathologies confondues, 2,8 éjaculats par patient ont été congelés avec 53 paillettes conservées en moyenne.

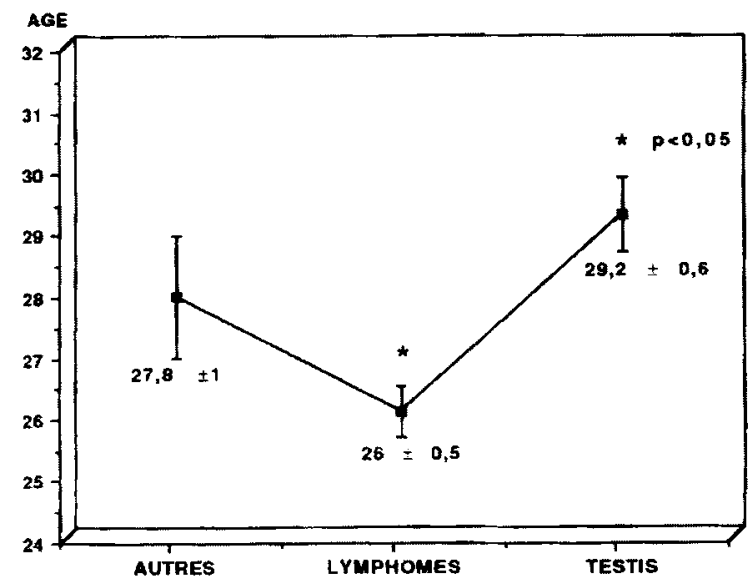

Figure 3 : Autoconservation :Age des patients en fonction des pathologies.

Le tableau 1 montre les résultats des caractéristiques spermatiques en fonction des pathologies. Les cancers testiculaires ont une numération $(\mathrm{p}<0,0001)$ et une mobilité $(\mathbf{p}<0,002)$ significativement plus faibles avant congélation et par conséquent un nombre de spermatozoïdes mobiles par paillettes significativement diminué. La numération des spermatozoïdes dans les tumeurs non séminomateuses est significativement inférieure à celle des séminomes $(\mathrm{p}<0,05)$.

Tableau 1 : Autoconservation : caractéristiques spermatiques avant et après congélation en fonction des pathologies $\left({ }^{*} p<0,001{ }^{* *} p<0,05\right)$.

\begin{tabular}{|c|c|c|c|c|c|c|}
\hline PATHOLOGIES & NUMERATION & $\begin{array}{c}\text { MOBILITE } \\
\text { AVANT }\end{array}$ & $\begin{array}{l}\text { MOBILITE } \\
\text { APRÉS }\end{array}$ & $\begin{array}{l}\text { N MOBILES / } \\
\text { PAILLETTES }\end{array}$ & VITALITE & $\begin{array}{c}\text { FORMES } \\
\text { NORMALES }\end{array}$ \\
\hline TOUS CANCERS CONFONDUS & $49,7 \pm 3,2$ & $44,4 \pm 1,5$ & $13,5 \pm 0,75$ & 0,83 & $74 \pm 0,9$ & $31,9 \pm 1,3$ \\
\hline AUTRES CANCERS & $66,2 \pm 8,5$ & $47,4 \pm 2,0$ & $15,2 \pm 1,8$ & 1,25 & $76,5 \pm 2,3$ & $33,3 \pm 3,2$ \\
\hline LYMPHOMES & $69,5 \pm 5,6$ & $44,5 \pm 1,5$ & $13,7 \pm 1,1$ & 1,19 & $73,2 \pm 1,3$ & $33 \pm 1,9$ \\
\hline K TESTIS & $30,4 \pm 2,7^{*}$ & $39,4 \pm 1,5^{*}$ & $12,3 \pm 1,3$ & $0,46^{*}$ & $75,3 \pm 1,4$ & $29,8 \pm 2,2$ \\
\hline $\begin{array}{l}\text { TUMEURS NON SEMINOMATEUSES } \\
\text { SEMINOMES } \\
\text { NON PRÉCISES }\end{array}$ & $\begin{array}{c}23,3 \pm 3^{* *} \\
34,3 \pm 5,5^{* *} \\
31,6 \pm 4,5\end{array}$ & $\begin{array}{l}38,8 \pm 3,4 \\
40,7 \pm 2,9 \\
38,8 \pm 2,1\end{array}$ & $\begin{array}{c}9,9 \pm 1,9 \\
15,9 \pm 2,8 \\
11,3 \pm 2\end{array}$ & $\begin{array}{l}0,26^{*} \\
0,68 \\
0,44\end{array}$ & $\begin{array}{l}73,7 \pm 3,9 \\
76,5 \pm 2,7 \\
75,9 \pm 1,7\end{array}$ & $\begin{array}{c}28,1 \pm 4,1 \\
26,5 \pm 3 \\
33,8 \pm 3,9\end{array}$ \\
\hline
\end{tabular}



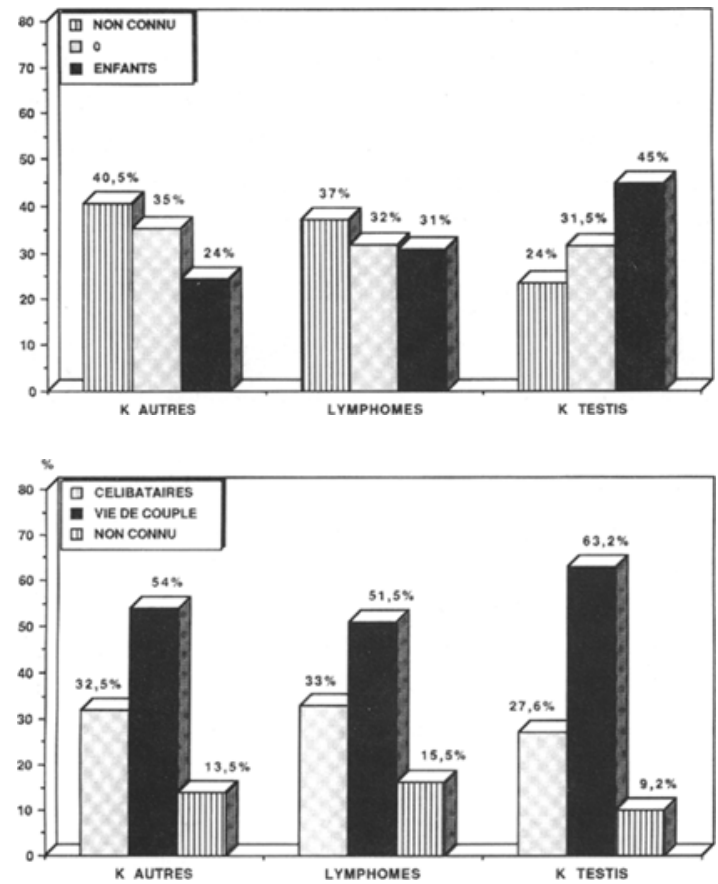

Figure 4: Autoconservation : Situation maritale et paternité des patients en fonction des pathologies.

Par contre le taux de récupération de la mobilité après congélation n'est pas différent selon les pathologies : $30 \%$ en moyenne.

\section{Devenir du sperme mis en garde}

L'analyse au 1/01/95 montre que 27 couples/210 ont utilisé des paillettes $(12,8 \%$ vs $10,6 \%$ enquête nationale), $20 \%$ ont demandé la destruction et pour $72,8 \%$ il y a poursuite de la garde (même après utilisation dans certains cas). Mais dans $44 \%$ des cas cette poursuite est une décision unilatérale du CECOS pour des patients perdus de vue ou ne répondant pas aux relances.

\section{Résultats de l'utilisation des paillettes (Tableau 2)}

- Toutes techniques confondues 12 grossesses ont été obtenues en lère demande (TMSC $=9 \%$ ) et 6 en deuxième demande (TMSC $=12,5 \%$ ). 1/3 des couples ont bénéficié successivement de plusieurs tech-
Tableau 2 : Autoconservation : résultats de l'utilisation des paillettes en fonction des

\begin{tabular}{|c|c|c|c|}
\hline METHODES & IC & IU & FIV \\
\hline NOMBRE DE COUPLES & 27 & 10 & 13 \\
\hline NOMBAE DE CYCLES & 124 & 29 & 27 \\
\hline GROSSESSES & 13 & 2 & 3 \\
\hline NOMBAE MOYEN DE CYCLES & 3 & 2,60 & 2,90 \\
\hline$\%$ SUCCES PAR CYCLE & 10,5 & 6,9 & 11,1 \\
\hline$\%$ SUCCES PAR COUPLE & 48,2 & 20 & 48,1 \\
\hline
\end{tabular}

niques en première demande et $1 / 4$ en deuxième demande.

- 21 inséminations intracervicales ont été tentées d'emblée. Le nombre de spermatozoïdes mobiles est significativement plus élevé dans les couples qui ont obtenu une grossesse $(2,56$ vs 1,58 millions $)$ $(\mathrm{p}<0,01)$.

Le nombre de spermatozoïdes mobiles pour le sperme utilisé en IU et en FIV est significativement plus faible avec des écarts de valeurs importants $(0,16$ à 3,15 Millions $)$.

\section{Suivi de la fertilité ultérieure des patients}

Notre étude a montré que $44 / 210$ soit 1 patient sur 5 a contrôlé son spermogramme après le traitement.

\section{DISCUSSION}

Ces résultats montrent que le nombre de patients adressés au CECOS pour autoconservation de sperme est en augmentation constante. Les progrès de l'assistance médicale à la procréation et notamment l'injection intracytoplasmique de spermatozoïdes dans l'ovocyte (ICSI) a totalement modifié les critères de conservation et incite les biologistes à congeler tous les spermes quelle que soit leur qualité. Il n'y a plus de limite inférieure de numération et la tendance actuelle est de tout conserver à partir du moment ou il y a des spermatozoïdes vivants, si bien que le pourcentage de spermes effectivement congelés par rapport aux demandes est en 
constante augmentation depuis 3 ans (97\% cette année en France).

L'âge, la situation matrimoniale et la paternité ne semble pas avoir d'influence sur la décision de conservation de sperme. Il faut noter toutefois que dans notre étude, les cancers testiculaires se singularisent par une moyenne d'âge plus élevée, fait non retrouvé dans l'enquête nationale multicentrique et à interpréter dans le cadre d'une enquête prospective sur un plus grand nombre de cas. Il n'a pas été noté dans notre série d'apparition plus précoce des cancers testiculaires malgré l'augmentation de l'incidence ces dernières années.

Les résultats de la conservation dépendent des caractéristiques initiales du sperme. L'un des mérites de l'autoconservation a été de permettre la mise en évidence des altérations de la spermatogenèse liées à la pathologie $[6,7,9]$ et ainsi de ne pas attribuer à posteriori une stérilité ou une hypofertilité antérieure uniquement au traitement. Cette série confirme que l'atteinte initiale du sperme est plus importante dans les cancers du testicule et plus particulièrement dans les tumeurs non séminomateuses que dans les autres cancers, notamment les lymphomes malgré les épisodes fébriles des Hodgkin. Toutefois la tolérance à la congélation des cancers testiculaires apparaît identique quant au \% de récupération de la mobilité. Le nombre de mobiles par paillettes dépend alors uniquement de la numération et de la mobilité de départ et non d'une susceptibilité plus importante au processus de congélation-décongélation.

Pour les paillettes et le choix de la technique d'assistance médicale à la procréation, il avait été décrit des seuils pour l'utilisation [4]. En présence de 2 millions de spermatozoïdes mobiles par paillette le taux de succès était identique à celui des femmes en IAD, en dessous de 0,5M aucun résultat n'était obtenu. L'avènement de la fécondation in vitro (FIV) avait permis de compléter les résultats obtenus en insémination Intra-Utérine. Dorénavant les techniques de fécondation assistée devraient permettre l'utilisation de spermes très pauvres. Les résultats d'AMP de notre série correspondent à une époque antérieure au 1/01/95 donc à l'utilisation de cette technique. Ces résultats sont cohérents avec ces seuils puisque les numérations des spermes ayant permis les grossesses en IAC sont supérieures à 2 Millions et les échecs de FIV à des spermes $<0,5$ millions. Quelle que soit la durée de la conservation, il n'y a pas de variation du taux de succès ce qui confirme des travaux antérieurs [4]. Le pourcentage d'utilisation est faible mais nous avons une grande proportion de patients perdus de vue ; la demande d'utilisation des paillettes souvent tardive et certains traitements anti cancéreux sont maintenant moins agressifs à long terme sur la spermatogenèse [9]. L'ICSI va peutêtre entraîner une augmentation des demandes à plus ou moins brève échéance.

Le grand problème reste notre incertitude quant à la récupération de la spermatogenèse. Les lésions de la spermatogenèse ne sont pas toutes irréversibles, mais les délais de récupération sont malheureusement difficiles à évaluer car une faible proportion de patients pratiquent un spermogramme de contrôle et nombreux sont les sujets perdus de vue ou qui ne répondent pas aux relances annuelles. Toutefois avant de programmer toute technique d'AMP un contrôle du spermogramme s'avère indispensable. Si celui-ci était fait systématiquement comme nous le proposons aux patients, nous aurions une notion plus précise de la fertilité ultérieure des patients en fonction des protocoles de traitement. La fédération nationale des CECOS à la suite de cette enquête rétrospective, a établi un dossier d'enquête prospective qui devrait permettre dans quelques années de répondre à nos interrogations. 


\section{CONCLUSION}

L'évolution des traitements anti-cancéreux permet d'obtenir de plus en plus de guérison. Mais prévoir la récupération de la spermatogenèse est difficile et varie avec le type du traitement et la susceptibilité propre des patients. A ce jour aucun protocole de prévention n'est encore applicable à l'homme, c'est pourquoi l'autoconservation de sperme doit être proposée à tout patient jeune en âge de procréer avant tout traitement potentiellement stérilisant. Plusieurs prélèvements de sperme sont nécessaires à 2-3 jours d'intervalle, 8 jours seulement sont à prévoir pour l'ensemble des déplacements. A cette occasion un contrat de garde est établi avec le patient précisant les modalités de conservation et d'utilisation éventuelle, et un spermogramme de contrôle un an après le traitement est proposé. La possibilité de préserver leur fertilité ultérieure a un impact psychologique important pour les patients. Elle leur permet de se projeter dans l'avenir et les conduit souvent, face à cet espoir de vie, à mieux accepter la lourdeur du traitement. Ne pas proposer cette autoconservation aux patients devant subir de tels traitements est un manquement grave à une obligation de moyens pour le médecin.

\section{REFERENCES}

1. AUROUX M. : Grossesses après chimiothérapie: risque génétique. Cahiers Oncol., 1992, $1: 2-34$.

2. COSTABILE R.A. : The effects of cancer and cancer therapy on male reproductive system. J. Urol.,1993, $149: 1327-1330$.

3. CZYGLICK F., KUNSTMANN J.M., JOUANNET P. : Autoconservation de sperme et preservation de la fertilité des hommes stérilisés. In Y. Englert, J.F. Guérin, P. Jouannet Eds. Stérilité masculine et procréations médicalement assistées. Paris, Douin, 1989 : 113-120.

4. CZYGLICK F. : Autoconservation de sperme avant thérapeutique stérilisante: indications et résultats. In CECOS ed, L'insémination artificielle, Paris, Masson, 1991 : 53-61.
5. FORMAN D., MOLLER H. : Testicular cancer. Cancer Surv., 1994, 19-20 : 323-341.

6. HANSEN P.V., GLAVIND K., PADURO J. et al. : Paternity in patients with testicular germ cell cancer: pretreatment and post treatment findings. Eur. J. Cancer, 1991, $27:$ 1385-1389.

7. HO G.T., GARDNER H., MOSTOFI K. et al. : The effects of testicular non germ cell tumors on local spermatogenesis. Fertil. Steril.,1994, 62 : 162-166.

8. HOERNI B., MAURIAC L. : Chimiothérapie anticancéreuse et troubles de la fertilité chez l'homme. Annal. Endocrinol. 1991 : 269-272.

9. MARMOR D., DUYCK F. : Male reproductive potential after MOPP therapy for Hodgkin disease : a long term survey. Andrologia, 1995, 27 : 99-106

10-MEIRON D., SCHENKER J.G. : Cancer and male fertility. Human Reprod., 1995, $10: 2017-2022$.

11. PALERMO G.D., COHEN J., AUKANI M. et al. : Intracytoplasmic sperm injection: a novel treatment for all forms of male factor infertility. Fertil. Steril., 1995, 63 : 1231-1236.

12. SANGER W.G., OLSON J.H., SHERMAN J.K. : Semen cryobanking for men with cancer: criteria change. Fertil. Steril., 1992, 58 : 1024-1027.

\section{ABSTRACT}

Semen Cryopreservation in malignant diseases : Retrospective study

\section{BARTHELEMY}

Cryopreservation of semen of patients with malignant diseases (testicular cancer, Hodgkin' disease and lymphoma) is a realistic option to preserve fertility of young patients before chemotherapy and/or radiation or sugery. We show here the results of a retrospective study in Cecos-Tours of cryopreservation between 1980 to 1990 and its outcome in 1995, in comparison with the national retrospective study of French Cecos Federation.

This survey demonstrates an increase in cryopreservation demands and in the \% of cryopreserved semen, especially in testicular cancer where semen alterations are significantly more important, because of the pro- 
gress in assisted reproductive technology, particularly the efficacy of ICSI in low spermatozoal densities.

In the absence of a real spermatogenesis protection in human, semen cryopreservation is an absolute indication before such treatments detrimental for fertility potential.

Key-words : Semen cryopreservation, cancer therapy, assisted reproductive technology, testicular cancer, Hodgkin' disease. 